\title{
Uncontrolled inflammation of the nervous system
}

Inherited CD59 deficiency Deniz Yuksel, MD, Kader Karli Oguz, MD, Ebru Azapagası, MD, Selman Kesici, MD, Busranur Cavdarli, MD,
Bahadir Konuskan, MD, and Haluk Topaloglu, MD

Neurology: Clinical Practice October 2018 vol. 8 no. 5 e18-e20 doi:10.1212/CPJ.0000000000000511

\author{
Correspondence \\ Dr. Topaloglu \\ htopalog@hacettepe.edu.tr
}

CD59 regulates the complement activation cascade at the final step by inhibiting formation of membrane attack complex. ${ }^{1}$ The lack of CD59 allows uncontrolled complement amplification following spontaneous, viral, or postviral induced complement activation. ${ }^{2}$ Inherited CD59 deficiency is a rare autosomal recessive disorder characterized by chronic hemolysis, early-onset recurrent peripheral neuropathy resembling Guillain-Barré syndrome or chronic inflammatory demyelinating polyneuropathy, and together with recurrent strokes. ${ }^{3-6}$

\section{PRACTICAL IMPLICATIONS}

Eculizumab may be effective in reversing neurologic symptoms of inherited CD59 deficiency.

We describe a family with isolated CD59 deficiency with peculiar findings: acute-onset immune-mediated cerebellitis, hemorrhagic longitudinal myelitis, acute disseminated encephalomyelitis (ADEM)-like demyelination in CNS, and subclinical mild chronic neuropathy. This report extends the clinical spectrum of inherited CD59 deficiency syndrome.

Patient 1 is an 11-year-old girl who presented with acute fever, vomiting, and fatigue within a day. The pedigree is presented in figure e-1 (links.lww.com/CPJ/A44). Her father had a form of acute peripheral neuropathy beginning at age 28 years and died shortly after the onset of the disease. Her aunt could not walk after a febrile illness, later developed scoliosis, and died at age 16 years. Another aunt died with presumed meningitis at 3 months of age. One sibling was admitted to the hospital a few days before patient 1's admission (see patient 2). Twelve years prior, her sister had died due to rapidly ascending neurologic signs at age 13 months.

On admission, patient 1 had dysarthric speech, horizontal nystagmus, dysmetria, and ataxic gait. There was pes cavus and thenar atrophy. She could only walk 10 meters.

The patient was admitted with a diagnosis of acute cerebellitis. Initial laboratory values were white blood cell count $8,350 / \mu \mathrm{L}$ (69\% neutrophils), hemoglobin $11 \mathrm{~g} / \mathrm{dL}$, platelet count $204 \times$ $10^{3} / \mu \mathrm{L}$, C-reactive protein $116 \mathrm{mg} / \mathrm{L}$. CSF examination showed abundant leukocytes $(2,000 /$ $\mathrm{mm}^{3}$ ), protein $450 \mathrm{mg} / \mathrm{dL}$, glucose $54 \mathrm{mg} / \mathrm{dL}$ (blood glucose $114 \mathrm{mg} / \mathrm{dL}$ ), with normal culture. MRI at presentation revealed diffuse cerebellar cortical edema with pial enhancement suggestive of acute cerebellitis, accompanied by diffuse linear enhancement of the cauda equina. Additional T2 hyperintense lesions in bilateral middle cerebellar peduncles were present (figure 1). She was started on IV immunoglobulin. She partially responded with improvement of ataxia and nystagmus after the fifth dose. Oral prednisolone therapy was added. Two days later, hypertension accompanied by seizures and nystagmus developed once again. MRI 5 days later shows pial and ependymal enhancement. Cortical and subcortical lesions at bilateral occipital and frontoparietal lobes were noticed on T2-weighted imaging. Simultaneous thoracic spinal MRI shows myelopathy by $\mathrm{T} 2$ hyperintensity involving both gray and white matter of the cord at the T7-T9 level (figure e-2, links.lww.com/CPJ/A44). Furthermore, thenar atrophy, mild pes cavus, and hypoactive deep tendon reflexes were suggestive of a neuropathic disorder. Three months into the diagnostic journey, CD59 deficiency was detected by flow cytometry.

Department of Pediatric Neurology (DY) and Pediatric Intensive Care Unit (EA, SK), University of Health Sciences, Dr. Sami Ulus Maternity and Children's Health and Diseases Training and Research Hospital; Departments of Radiology (KKO) and Pediatric Neurology (BK, HT), Faculty of Medicine, Hacettepe University; and Ankara Numune Training and Research Hospital (BC), Genetic Diseases Diagnosis Center, Ankara, Turkey.

Funding information and disclosures are provided at the end of the article. Full disclosure form information provided by the authors is available with the full text of this article at Neurology.org/cp. 

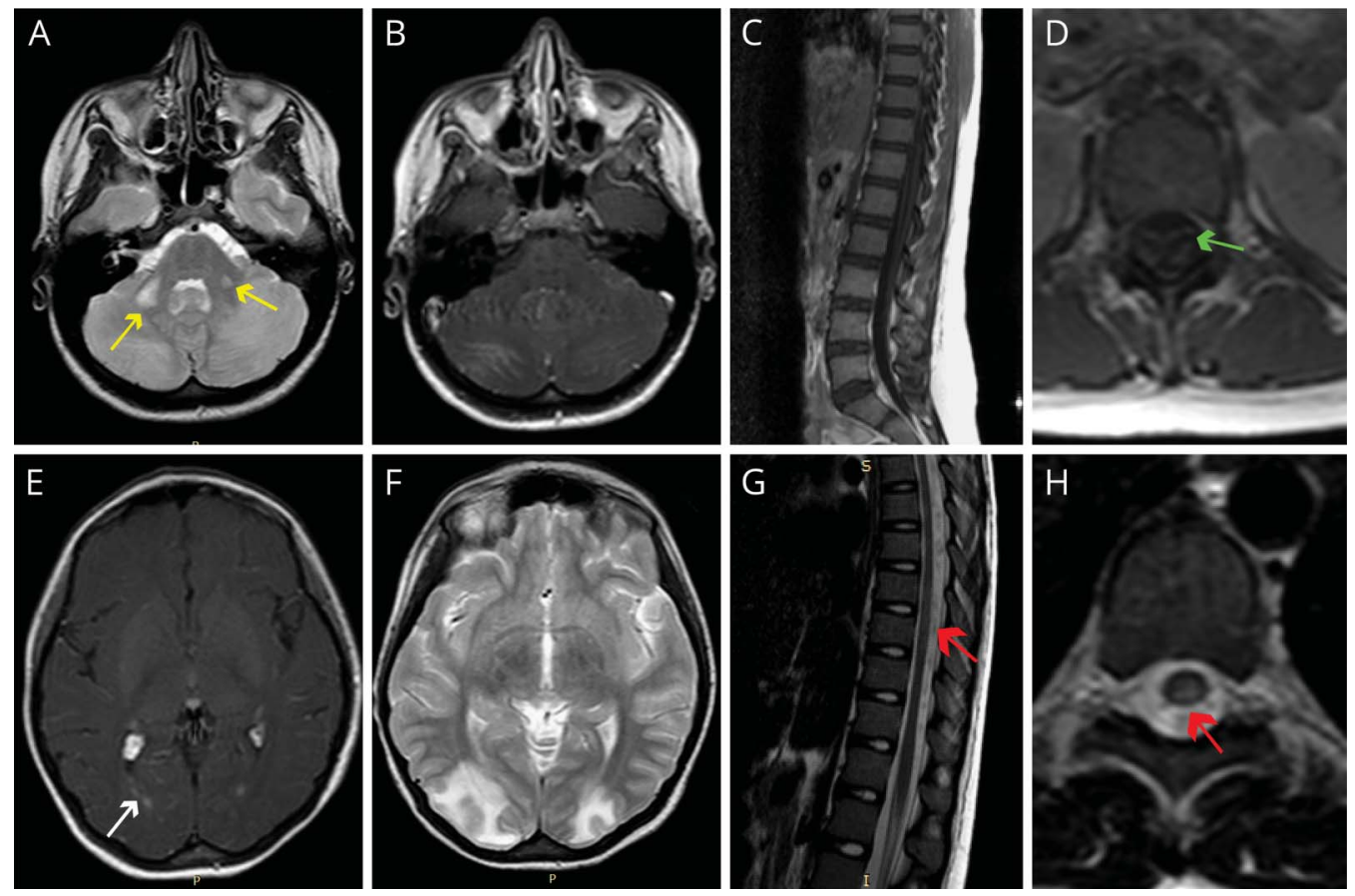

MRI at the time of presentation reveals diffuse cerebellar cortical edema (A; yellow arrows) with pial enhancement (B) suggestive of acute cerebellitis, accompanied by diffuse linear enhancement of the cauda equina (C, D; green arrow). Additional T2-hyperintense lesions in bilateral middle cerebellar peduncles are shown (A). Although there is no supratentorial meningeal enhancement at initial MRI (not shown), an MRI at 5 days later shows pial and ependymal enhancement (E; white arrow). Cortical and subcortical lesions at bilateral occipital (F) and frontoparietal lobes (not shown) consistent with "posterior reversible encephalopathy syndrome" are seen on T2-weighted imaging. Simultaneous thoracic spinal MRI shows myelopathy by T2 hyperintensity involving both gray and white matter of the cord at the T7-T9 level ( $G, \mathrm{H}$; red arrows).

CD59 mutation analysis revealed a homozygous missense mutation (c.146A>T[p.Asp49Val]). Based on previous reports, the patient was put on eculizumab. Within 7 months of onset, she gradually improved with no ataxia and full ambulation at the last visit. She continues to take this drug according to the scheme.

Patient 2, the younger sibling of patient 1 , presented at age 10 years with acute urinary incontinence and walking difficulty. On examination, he had lower extremity weakness. One day later, the patient's consciousness decreased and neck stiffness developed. In the CSF, there were 25 leukocytes $/ \mu \mathrm{L}$, and protein was elevated at $70 \mathrm{mg} / \mathrm{dL}$. This boy's MRI showed T2-hyperintense lesions in bilateral middle cerebellar peduncles and right cerebellar hemisphere with many additional lesions in the deep and peripheral cerebral white matter. From cervical level 3 down to the conus medullaris, profound acute myelopathy and radiculopathy with hemorrhage were present on T1 (figure e-2, links.lww.com/CPJ/A44). The boy received IV steroids, plasma exchange, erythrocyte infusions, and life support. He died on the 86th day of admission. The CD59 mutation analysis of this patient was identical to that of his sister, which was shown postmortem.

\section{Genetic analyses}

CD59 gene sequence analysis was performed with MiSeq nextgeneration sequencing platform (Illumina, San Diego, CA).
Genomic DNA was isolated from peripheral lymphocytes according to the manufacturer's standard procedure with Anatolia magnetic bead kit (Anatolia, Turkey). Coding exons and flanking regions were amplified with in-house designed PCR primers, designed with Primer Designer v.2.0 (Scientific \& Educational Software program, Denver, CO) software. Library preparation was carried out with the Nextera XT kit (Illumina). Sequences were aligned to the $\mathrm{hg} 19$ genome within MiSeq Reporter software (Illumina) and visualized with IGV 2.3 (Broad Institute, Cambridge, MA) software.

As shown in figure 1, the CD59 mutation analysis in patients 1 and 2 showed a homozygous deletion (c.146A $>\mathrm{T}$ [p.Asp49Val]). Heterozygous deletion was detected in their mother's analyses. The parents are not relatives; rather, they are individuals from the same village.

\section{Discussion}

Mutations in CD59 leading to loss of function have been described previously in 12 patients, all of whom had neurologic findings. Eleven of 12 patients presented with recurrent peripheral neuropathy while 6 of them presented with recurrent strokes. ${ }^{2}$ The age at onset of the first symptoms of previously described cases was generally reported during infancy. ${ }^{2-8}$ Our cases presented with acute neurologic features at a later age. However, the examination findings were 
consistent with chronic neuropathy, suggesting that both cases actually may have had symptoms before the acute presentation.

Remarkably, caudal involvement and middle cerebellar peduncle lesions were also present in previously reported patients. $^{3,4,8}$ Previously unknown longitudinal diffuse hemorrhagic myelopathy, ADEM-like cerebral demyelinating patchy lesions, and acute cerebellitis findings were detected by neuroimaging. Different from the cases in the literature, cerebrovascular events were not present. We follow 4 other patients who are treated with eculizumab. There have been no new neurologic insults (unpublished clinical observations). At this stage, eculizumab therapy was initiated in the index case. After 5 months with eculizumab ( 7 months into the symptoms), her ataxia has resolved completely.

\section{Author contributions}

D. Yuksel: acquisition of data and interpretation. K.K. Oguz: acquisition of data and interpretation. E. Azapagası: acquisition of data. S. Kesici: acquisition of data. B. Cavdarli: acquisition of data. B. Konuskan: acquisition of data. H. Topaloglu: acquisition of data and interpretation.

\section{Acknowledgment}

The authors thank Prof. Banu Anlar and the Faculty of Pediatric Neurology at the Dr. Sami Ulus Hospital).

\section{Study funding}

No targeted funding reported.

\section{Disclosure}

D. Yuksel, K.K. Oğuz, E. Azapagasi, S. Kesici, B. Cavdarlı, and B. Konuşkan report no disclosures. H. Topaloglu receives research support from PTC Therapeutics and GlaxoSmithKline. Full disclosure form information provided by the authors is available with the full text of this article at Neurology.org/cp.

Received March 1, 2018. Accepted in final form May 29, 2018.

\section{References}

1. Sugita Y, Masuho Y. CD59: its role in complement regulation for therapeutic use. Immunotechnology 1995;1:157-168.

2. Tabib A, Karbian N, Mevorach D. Demyelination, strokes, and eculizumab: lessons from the congenital CD59 gene mutations. Mol Immunol 2017;89:69-72.

3. Nevo Y, Ben-Zeev B, Tabib A, et al. CD59 deficiency is associated with chronic hemolysis and childhood relapsing immune-mediated polyneuropathy. Blood 2013; 121:129-135.

4. Haliloglu G, Maluenda J, Sayinbatur B, et al. Early-onset chronic axonal neuropathy, strokes, and hemolysis: inherited CD59 deficiency. Neurology 2015;84:1220-1224.

5. Ben-Zeev B, Tabib A, Nissenkorn A, et al. Devastating recurrent brain ischemic infarctions and retinal disease in pediatric patients with CD59 deficiency. Eur J Paediatr Neurol 2015;19:688-693.

6. Ardicli D, Taskiran EZ, Kosukcu C, et al. Neonatal-onset recurrent Guillain-Barre syndrome-like disease: clues for inherited CD59 deficiency. Neuropediatrics 2017;48: 477-481.

7. Mevorach D, Reiner I, Grau A, et al. Therapy with eculizumab for patients with CD59 p.Cys89Tyr mutation. Ann Neurol 2016;80:708-717.

8. Höchmann B, Dohna-Schwake C, Kyrieleis HA, Pannicke U, Schrezenmeier H. Targeted therapy with eculizumab or inherited CD59 deficiency. N Engl J Med 2014; 370:90-92. 